\title{
EFFECTS OF HEAVY-ELEMENT ABUNDANCE ON CLASSIFICATION OF G-TYPE GIANTS
}

\author{
P. C. KEENAN
}

Perkins Observatory, Ohio State and Ohio Wesleyan Universities, U.S.A.

\begin{abstract}
Five stars, including $\zeta$ Cyg, which had been classified as G6 II to G8 II bright giants, were found by $\mathrm{O}$. C. Wilson to have $\mathrm{K}$-line luminosities close to those of class III giants. These stars show enhancement of the lines of $\mathrm{Ba}$ II and other heavy-metal ions also. To eliminate the serious effect of this abundance anomaly on the spectroscopic luminosities new luminosity criteria involving only intercomparisons of lines of elements of the fourth period ( $\mathrm{Ti}, \mathrm{Fe}$, etc.) were applied on $9 \AA \mathrm{mm}^{-1}$ Coudé spectrograms taken by $\mathrm{O}$. C. Wilson. This Coudé classification gave luminosity classes near IIla for these stars, implying absolute magnitudes considerably below those of bright giants but somewhat above Wilson's $\mathbf{M}_{\mathbf{K}}$ values.

Another advantage of Coudé classification is the possibility of estimating luminosities for individual barium stars. From Wilson's plate of HD 205011 a luminosity class of III-IIIa is derived. This is consistent with the mean value of $M_{\boldsymbol{v}}=-0.4$ derived from statistical parallaxes by MacConnell, Frye and Upgren (Astron. J. 77, (1972), 384) for the barium stars.
\end{abstract}

The detailed account of this investigation will be published elsewhere.

\section{DISCUSSION}

Williams: Do you have any comment on the luminosity of the prototype BaII star $\zeta$ Cap, which appears from Wilson's $M_{v}(\mathrm{~K})$ to be very luminous?

Keenan: I have no spectrograms of this star, and have no reason to think that it may not be as bright as $M_{v} \approx-3$. 